\title{
A INTRODUÇÃO E DISTRIBUIÇÃO DO TUCUNARÉ-AZUL E DO TAMBAQUI NO PANTANAL CORUMBAENSE
}

\section{THE INTRODUCTION AND DISTRIBUTION OF BLUE PEACOCK BASS AND TAMBAQUI IN THE PANTANAL OF CORUMBÁ}

\author{
Renner Fernando da Silva Córdova Junior ${ }^{1}$
}

Submetido: 12/01/2021 / Aprovado: 26/10/2021 / Publicado: 30/11/2021.

\begin{abstract}
Resumo
O Pantanal possui características únicas, porém similares à Bacia Amazônica, principalmente no que tange os ciclos de cheias em ambas as regiões. Tais semelhanças permitiram que espécies de peixes introduzidas como o tucunaré-azul (Cichla piquiti) e o tambaqui (Colossoma macropomum) se desenvolvessem na região pantaneira após escape de tanques, escavados na década de 1980. Neste sentido, foi realizada a averiguação da ocorrência de tais espécies nos principais pontos de pesca, no rio Paraguai, partindo da cidade de Corumbá, na planície pantaneira. Nos pontos analisados, foi feita pesca de exemplares com a utilização de apetrechos de pesca permitidos pela legislação vigente para a pesca amadora, considerando o esforço de pesca de oito horas por dia de campanha amostral, totalizando sete campanhas e 56 horas de esforço amostral entre 2018 e 2019. Os resultados apontam que o tucunaré já se encontra amplamente espalhado na planície pantaneira, já o tambaqui ainda se mantém restrito à algumas baías. Sendo assim, é necessário que haja um monitoramento das espécies e estudos comportamentais desses espécimes para compreender e mitigar os impactos da sua introdução em tão importante planície.
\end{abstract}

Palavras-chave: Peixes amazônicos. Rio Paraguai. Invasão.

\begin{abstract}
The Pantanal has unique characteristics, but they are similar to the Amazon Basin, especially with regard to flood cycles in both regions. Such similarities allowed introduced fish species such as the blue peacock bass (Cichla piquiti) and the tambaqui (Colossoma macropomum) to develop in the Pantanal region after escaping from excavated tanks in the 1980s. In this sense, the investigation of the occurrence of such species was carried out in the main fishing spots, on the Paraguay River, starting from the city of Corumbá in the Pantanal plain. At the analyzed points, specimen fishing was carried out using fishing equipment allowed by the current legislation for amateur fishing, considering the fishing effort of eight hours per day of the sampling campaign, totaling seven campaigns and 56 hours of sampling effort between 2018 and 2019. The results show that the peacock bass is already widely spread on the Pantanal plain, while the tambaqui is still restricted to some bays. Therefore, it is necessary to monitor the species and conduct behavioral studies to understand and mitigate the impacts of their introduction on such an important plain.
\end{abstract}

Keywords: Amazonian fish. Paraguay river. Invasion

\footnotetext{
${ }^{1}$ Msc. em Meio Ambiente e Desenvolvimento Regional. IFMS - Instituto Federal de Mato Grosso do Sul, campus Naviraí. Rua Hilda, 203, Bairro Boa Vista Naviraí, MS, Brasil. 79950-000. E-mail: renner.cordova@ifms.edu.br
} 


\section{INTRODUÇÃO}

O Pantanal é uma extensa planície sedimentar de relevo uniforme com morrarias que podem alcançar de 200 a 1000 metros de altitude (BRITSKI et al., 2007). Essa planície está situada na região Centro-Oeste do Brasil com uma área que abrange os estados de Mato Grosso e Mato Grosso do Sul, e possui um regime hidrológico bem definido em que os rios alternam um ciclo de cheia e seca na planície. Sua área varia entre 133.465 a 168.000 km2 (GALDINO et al., 2002; VILA da SILVA, 1995).

Ademais, o fluxo das águas dos rios exerce influência direta sobre a planície, pois dita a distribuição de recursos, habitats e da biodiversidade associada (ALHO, 2012). Dentro desse sistema, o rio Paraguai atravessa toda a planície e recebe, no caminho, rios tributários desde o seu nascimento no Planalto de Mato Grosso e drena todo o Pantanal até seu deságue no rio Paraná, na Argentina. Por essa razão, ele é considerado um dos principais rios (BRAZIL, 2014). Esse conjunto de caminhos aquáticos constitui a Bacia do Alto Paraguai (BAP).

Dentro dessa planície alagada, Corumbá é um dos principais municípios do estado de Mato Grosso do Sul, situado no extremo Oeste, à margem do rio Paraguai, com territórios limítrofes com o Paraguai e a Bolívia. A cidade possui vastos recursos naturais e a atividade pesqueira profissional é uma das principais fontes de renda para muitos indivíduos que realizam a chamada pesca artesanal ou amadora turística (MARIANI e ARRUDA, 2009).

$\mathrm{Na}$ década de 1980, peixes da Bacia Amazônica, como o tucunaré (Cichla piquiti) e o tambaqui (Colossoma macropomum), foram criados em tanques escavados em fazendas às margens dos rios Piquiri e Itiquira no alto Paraguai, atual estado de Mato Grosso, e com as cheias dos rios, os açudes foram inundados e esses peixes escaparam, acidentalmente, para o Pantanal (SÚAREZ et al., 2001; RESENDE et al., 2008; CÓRDOVA JUNIOR, 2020).

É importante ressaltar a diferença entre uma espécie introduzida da espécie exótica. A espécie introduzida é aquela que se encontra fora da região geográfica original. Já a espécie exótica provém de outros países ou alóctone ou quando é originária de outra região, bioma ou bacia, do mesmo país (ROSENFIELD e MANN, 1992). A introdução é entendida como a inserção de uma espécie nova em uma região, e ainda que sejam exóticas ou alóctones, essa inserção não apresenta distinções ecológicas consistentes. (AGOSTINHO e JÚLIO JUNIOR, 1996). Para Britski (1994), a introdução de espécies, sobretudo piscívoras, como é o caso do tucunaré, tem resultado em severos prejuízos à ictiofauna local, devido, principalmente, à atividade predatória da espécie introduzida e/ou pela transmissão de organismos patogênicos.

Pertencentes à família Cichlidea dentro da ordem Perciformes, o gênero Cichla agrupa peixes vorazes com alta taxa de predação e sua introdução pode ameaçar seriamente os peixes nativos, comprometendo a diversidade da assembleia ou mesmo causando a completa extinção regional de várias espécies (PELICICE e AGOSTINHO, 2009).

Atualmente são conhecidas 15 espécies de tucunarés, mas apesar da sua ocorrência natural ser a Bacia Amazônica, exemplares do gênero Cichla são encontrados em todo o território brasileiro (KULLANDER e FERREIRA, 2006; LANGEANI et al., 2007; WINEMILLER, 2001). A introdução dessa espécie é feita com a intensão de controlar outras espécies de peixes ou decorre acidentalmente por transbordamento dos tanques de piscicultura quando estes são confeccionados próximos a rios com fluxo de grandes cheias (SILVA et al., 1980; SÚAREZ et al., 2001). Introduções propositais para fins de peixamento e promoção do turismo de pesca também são relatadas em diversos estados brasileiros, sobretudo no nordeste e sudeste (ARAUJO et al., 2012).

Já o tambaqui (Colossoma macropomum) é um peixe pertencente à ordem Characiformes dentro da família Serrasalminae (BUCKUP et al., 2007). É considerado um peixe de grande porte, podendo alcançar $100 \mathrm{~cm}$ de comprimento e $30 \mathrm{~kg}$ de peso. É um peixe forrageiro onívoro que está 
associado com a dispersão de diversas plantas frutíferas que compõem a vegetação ciliar (PENNA et al., 2005; MACCORD et al.,2007; SOARES, 2007). No Pantanal, esse papel é desempenhado por várias espécies, sendo a mais significativa o pacu (Piaractus mesopotamicus). Ambas as espécies têm os hábitos alimentares semelhantes, o que pode aumentar a competição interespecífica por recursos (RODRIGUES, 2014; BRITSKI et al., 2007).

Súarez et al. (2001) alegaram que os peixes introduzidos se manteriam restritos às baias de águas mais claras ao norte do Rio Paraguai, contudo Resende et al. (2008) levantaram a possibilidade das espécies introduzidas se espalharem por todo o Rio Paraguai. Este trabalho sintetiza a introdução da espécie amazônica Cichla piquiti bem como a possível introdução do Colossoma macropomum e traz a dispersão atual dessas espécies no Pantanal Sul-Mato-Grossense bem como os principais pontos para a captura dessas espécies, partindo do município de Corumbá, e comenta alguns possíveis impactos dessas espécies na região Pantaneira.

\section{MATERiAl E MÉTOdos}

\subsection{Caracterização da área de estudo}

O rio Paraguai é o principal eixo de drenagem que corta o Pantanal e une, dentre outras, as cidades de Cáceres, no Mato Grosso, e Corumbá, no Mato Grosso do Sul. Esse rio recebe maior porção de água das chuvas de janeiro a março no alto Pantanal (Cáceres, MT), drenando bacias desde o planalto de entorno e escoando para a calha do rio (SABINO, 2015). Como se fosse uma onda, lentamente, a enchente só alcança Corumbá num período de dois a três meses depois, quando cessa o período chuvoso (BRITSKI et al., 2007). Durante essa época, o rio Paraguai se comporta como uma larga faixa de água que vai se escoando lentamente rumo ao Sul, demorando até seis meses para deixar o território brasileiro (CARVALHO, 1986). Dentro dessa grande planície de inundação, além dos leitos dos rios e afluentes, aparecem as grandes lagoas, corixos, vazantes e baías que se conectam temporariamente ou permanentemente ao rio Paraguai (SABINO, 2015).

Partindo do porto Geral, na cidade de Corumbá, foram escolhidas regiões de pesca (baías e corixos) ao note e ao sul que se conectam ao rio Paraguai. Tais regiões (Tabela 1) são conhecidas dos pescadores locais que relatam, de forma popular, a ocorrência de espécies exóticas nessas regiões. 
Tabela 1 - Locais escolhidos para averiguação da ocorrência de tucunaré e tambaqui na região de Corumbá, Mato Grosso do Sul.

\begin{tabular}{|c|c|c|c|}
\hline \multirow{4}{*}{ Norte* } & Pontos & $\begin{array}{l}\text { Coordenadas } \\
* * *\end{array}$ & Datas \\
\hline & 1-Cervo Paraguai-mirim & $\begin{array}{l}18^{\circ} 30^{\prime} 05,01 " \mathrm{~S} \\
57^{\circ} 18^{\prime} 48,04 ” \mathrm{~W}\end{array}$ & $20 / 11 / 2018$ \\
\hline & 2-Baía Vermelha & $\begin{array}{l}18^{\circ} 25^{\prime}, 42.63 ” \mathrm{~S} \\
57^{\circ} 28^{\prime} 19.65^{\prime \prime} \mathrm{W}\end{array}$ & $21 / 11 / 2018$ \\
\hline & 3-Baía do Tuiuiú & $\begin{array}{l}18^{\circ} 48^{\prime}, 32.29^{\prime \prime} \mathrm{S} \\
57^{\circ} 39^{\prime} 17.15^{\prime \prime} \mathrm{W}\end{array}$ & 06/04/2019 \\
\hline \multirow{4}{*}{ Sul** } & 4-Baía Albuquerque & $\begin{array}{l}19^{\circ} 25^{\prime} 45.53 ” \mathrm{~S} \\
57^{\circ} 22^{\prime} 39.56 ” \mathrm{~W}\end{array}$ & $19 / 04 / 2019$ \\
\hline & 5-Baía da Galinha & $\begin{array}{l}19^{\circ} 42^{\prime} 19,4^{\prime \prime} \mathrm{S} \\
57^{\circ} 32^{\prime} 49,2^{\prime \prime} \mathrm{W}\end{array}$ & $12 / 07 / 2019$ \\
\hline & 6-Baía Grande & $\begin{array}{l}19^{\circ} 44^{\prime} 32.04^{\prime \prime} \mathrm{S} \\
57^{\circ} 35^{\prime} 54.46^{\prime \prime} \mathrm{W}\end{array}$ & $11 / 07 / 2018$ \\
\hline & $\begin{array}{l}\text { 7-Baía da Santa (Forte } \\
\text { Coimbra) }\end{array}$ & $\begin{array}{l}19^{\circ} 55^{\prime} 38.77^{\prime \prime} \mathrm{S} \\
57^{\circ} 50^{\prime} 49.52^{\prime \prime} \mathrm{W}\end{array}$ & $28 / 10 / 2018$ \\
\hline
\end{tabular}

*Regiões espalhadas em um percurso aproximado de $100 \mathrm{~km}$ subindo o rio Paraguai, partindo do porto geral de Corumbá. **Regiões espalhadas em um percurso aproximado de $150 \mathrm{~km}$, partindo do porto geral de Corumbá. *** Coordenada SUL (S), Coordenada OESTE (W).

\subsection{Amostragem}

Nos locais escolhidos (Tabela 01) foram realizadas sete campanhas amostrais entre $2018 \mathrm{e}$ 2019. Apesar do projeto estar amparados por licença de pesca amadora e pela licença para pesca científica $\mathrm{n}^{\circ}$ 62781-1, autorizado pelo Sistema de Autorização e Informação em Biodiversidade SiSBIO, não houve o depósito de exemplares em coleções nem a dissecação de exemplares. Cada ponto foi marcado por GPS modelo Garmin eTrex 10 e as coordenadas convertidas para o sistema de coordenadas geográficas com sistema SIRGAS 2000 para posterior construção de mapa em programa de georeferenciamento QGIS.

Nas mediações dos pontos demarcados, foi realizada a pesca esportiva de exemplares de tucunaré e tambaqui. Em cada campanha amostral, definiu-se um esforço amostral de oito horas, realizado pela equipe composta por três pessoas embarcadas em barco de alumínio de seis metros com propulsão de popa. Os apetrechos de pesca utilizados foram: varas, carretilhas, anzóis, iscas artificiais e naturais como a tuvira (Gymnotus ssp.) e o lambari (Astyanax ssp.), sendo que esse tipo de pesca se mostra mais eficaz para a captura das espécies de tucunarés nos mais variados ambientes (JEPSEN e WINEMILLER, 1997; GOMEIRO, 2010). Os mesmos apetrechos de pesca foram utilizados para a pesca do tambaqui, porém as iscas utilizadas foram iscas vivas, como caranguejo, minhocaçu, e frutos como a "laranjinha de pacu" (Pouteria glomerata) e goiaba.

\section{RESUltados E DiscuSsÃo}

A confirmação da ocorrência do tucunaré e do tambaqui se deu pela captura esportiva (captura seguida de devolução do peixe ao rio) em diversos pontos de pesca da região de Corumbá (Tabela 2). 
Tabela 2 - Levantamento das áreas de capturas de espécies introduzidas.

Localização

\begin{tabular}{ll}
\hline 1-Cervo (Paraguai-mirim) & Tucunaré-azul (Cichla piquiti) \\
& Tambaqui (Colossoma macropomum) \\
& Tambacu (híbrido)* \\
2-Baía Vermelha & Tambaqui (Colossoma macropomum) \\
& Tambacu (híbrido) \\
3-Baía do Tuiuiú & Tambaqui, (Colossoma macropomum), \\
& Tucunaré-azul (Cichla piquiti) \\
& Tambacu (híbrido)* \\
4-Baía Albuquerque & Tambaqui (Colossoma macropomum) \\
& Tucunaré-azul (Cichla piquiti) \\
5-Baía da Galinha & Tambacu (híbrido)* \\
\hline 6-Baía Grande & Tucunaré-azul (Cichla piquiti) \\
7-Baía da Santa (Forte Coimbra) & Tucunaré-azul (Cichla piquiti) \\
& Tambaqui, (Colossoma macropomum), \\
& Tucunaré-azul (Cichla piquiti) \\
& Tambacu (híbrido)*
\end{tabular}

* A ocorrência do híbrido é estimada a partir da captura do tambaqui, sendo necessárias maiores análises para o reconhecimento característico dos híbridos.

As espécies amazônicas, introduzidas no Pantanal, apresentam ampla distribuição pelo rio Paraguai, um dos principais rios da Bacia do Alto Paraguai (BAP) que drena a planície pantaneira. Os pontos de captura (Figura 1) ilustram as regiões de maior atividade pesqueira com ocorrência dessas espécies no município de Corumbá. 


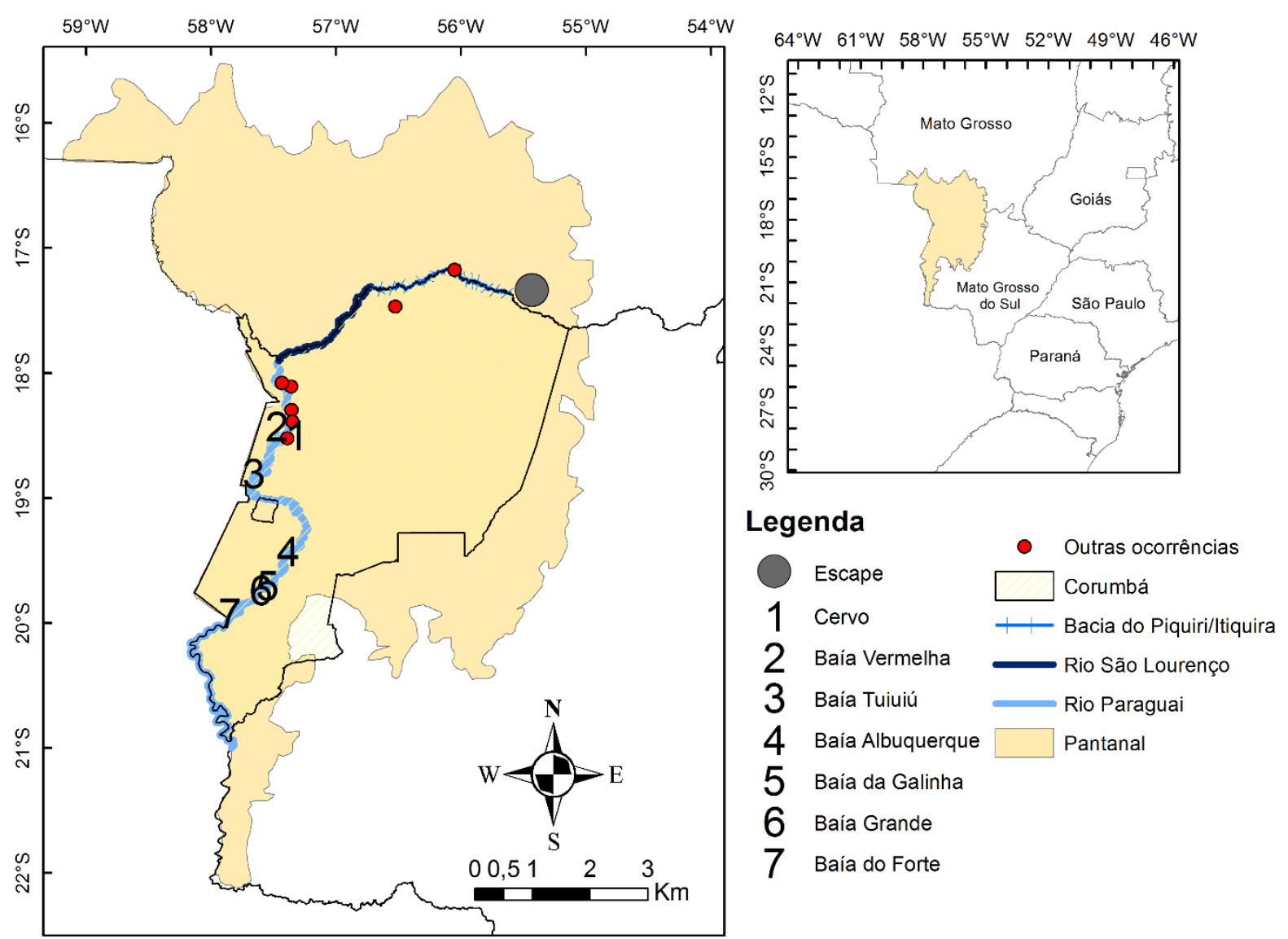

Figura 1. Distribuição de peixes amazônicos introduzidos no pantanal - Números indicam locais onde foram realizados os trabalhos de campo e os pontos relatos de ocorrência já publicados.

O tucunaré-azul, Cichla piquiti, tem sido pescado em diferentes regiões pantaneiras como na Baía do Chané, Baía do João, Baía do São Francisco, Baía São Pedro, Corixo Limoeiro, Cruz São Pedro, Corixo do Mata-Cachorro, Ilha do Coqueiro, rio Negrinho, rio Piquiri, rio São Lourenço, rio Paraguai-Mirim (MARQUES e RESENDE, 2005). Agora, sua pesca, também, está ocorrendo ao sul do rio Paraguai nas regiões da Baía Albuquerque, Baía da Galinha, Baía Grande e Baía Santa.

No final da década de 1970, o Pantanal entrava em seu maior ciclo de seca. Esse fenômeno alterou a ocupação humana e a extensão da criação de rebanhos bovinos (GALDINO et al., 2002). A seca possibilitou aumentar os rebanhos, mas para potencializar a criação do gado, foi necessária a criação de uma alternativa, de baixo custo, para obtenção de proteína animal que pudesse suprir as necessidades alimentares dos moradores das fazendas. Algumas fazendas, próximas à margem de rios da BAP, passaram a criar peixes amazônicos como fonte de proteína. O tucunaré possui acelerada taxa de evolução, com altos níveis de variação genética o que permitem fácil aclimatação e aumento populacional acelerado em condições certas (FARIAS et al., 1999). Já o tambaqui, por ser um peixe de grande porte e de grande resistência, oferece grande quantidade de proteína ao passo que sua alimentação é composta de alguns frutos de determinadas espécies vegetais que compõem a vegetação ciliar (PENNA et al., 2005; SOARES, 2007).

As condições ambientais da época favoreceram a introdução de diversos animais e plantas de maneira deliberada ou acidental, na região, que ocasionaram alterações ecológicas e ameaçaram a biodiversidade por modificaram o habitat e proporcionar o deslocando de espécies nativas (ALHO et al., 2011). Nessa época, espécies de peixes como o tucunaré-azul (Cichla piquiti) e o 
tambaqui (Colossoma macropomum) começaram a ser criados em lagos escavados às margens do rio Piquiri e Itiquira (SABINO, 2015).

\subsection{Introdução e dispersão}

No início da década de 1980, o Pantanal entrou em seu maior ciclo de cheia, sendo a maior delas em 1988 (GALDINO et al., 2002). Em 1986, as águas invadiram os tanques escavados nas margens dos rios Piquiri e Itiquira, favorecendo o escape dos espécimes criados para esses rios e posterior desague no rio Paraguai (SÚAREZ et al., 2001; RESENDE et al., 2008). As translocações de espécies nativas entre bacias hidrográficas, como no caso do tambaqui, tucunaré e pirarucu, que são espécies utilizadas nas pisciculturas com a finalidade de produzir proteína animal, é quase sempre acompanhado por escapes e resulta na introdução de espécies não nativas em ambientes naturais de praticamente todo o território nacional, com consequências negativas para o ambiente e para as espécies nativas (BRABO et al., 2016; ORSI e AGOSTINHO, 1999; VELLUDO et al., 2011).

Entre os anos de 1992 e 1994, Súarez et al. (2001), registraram a ocorrência de um peixe não nativo do gênero Cichla (popularmente como tucunarés) no Pantanal. A ocorrência foi confirmada nos rios Piquiri, Corrente e Itiquira, e em outros rios do Pantanal de Mato Grosso, totalizando 425 exemplares capturados durante aquele período.

Os exemplares coletados de tucunaré, na Bacia do Alto Paraguai, foram inicialmente identificados como Cichla monoculus, tucunaré-amarelo (LIPPARELLI, 1991). Entretanto, após a revisão taxonômica, a espécie foi identificada como sendo Cichla piquiti, o tucunaré-azul (RESENDE et al., 2008). A espécie não ficou restrita à bacia do rio Piquiri e se dispersou a jusante, para o rio Paraguai e alguns dos seus tributários, onde tem sido capturado principalmente na região do extenso município de Corumbá.

O tucunaré-azul é originado em ambientes lênticos, com águas quentes relativamente claras devido sua orientação visual (SABINO e ZUANON, 1998; WINEMILLER, 2001). Essas características são compatíveis com algumas regiões ao norte do Pantanal, e a previsão inicial era que tais características impediriam o espalhamento do tucunaré-azul para outras regiões pantaneiras, principalmente para a região sul (SÚAREZ et al., 2001). Contudo, o tucunaré-azul desceu e se espalhou por toda a planície pantaneira (Figura 1), conforme as previsões feitas no trabalho de Marques e Resende (2005) de que o tucunaré ocuparia a bacia pantaneira através do rio Paraguai.

Tanto o tucunaré-azul (Figura 2) e o tambaqui (Figura 3) acompanham o ciclo de cheia e seca que ocorre na planície Pantaneira. O ciclo de chuvas na região norte da BAP regula a quantidade de águas que serão drenadas para o rio Paraguai (GALDINO et al., 2002). As águas transportam e distribuem alimentos à medida que conectam baías e corixos ao rio Paraguai (BRITSKI et al., 2007). Essa dinâmica influencia a distribuição de diversas espécies de peixes endêmicas da região e proporciona o rearranjo das espécies introduzidas, favorecendo sua dispersão e instalação em trecho ou regiões que antes não ocorriam (MARQUES e RESENDE, 2005). Tais interações permitiram a integração das áreas de escape dessas espécies com o rio Paraguai e consequentemente sua distribuição em corixos e baías propícias ao estabelecimento de tais espécies nas últimas décadas. 


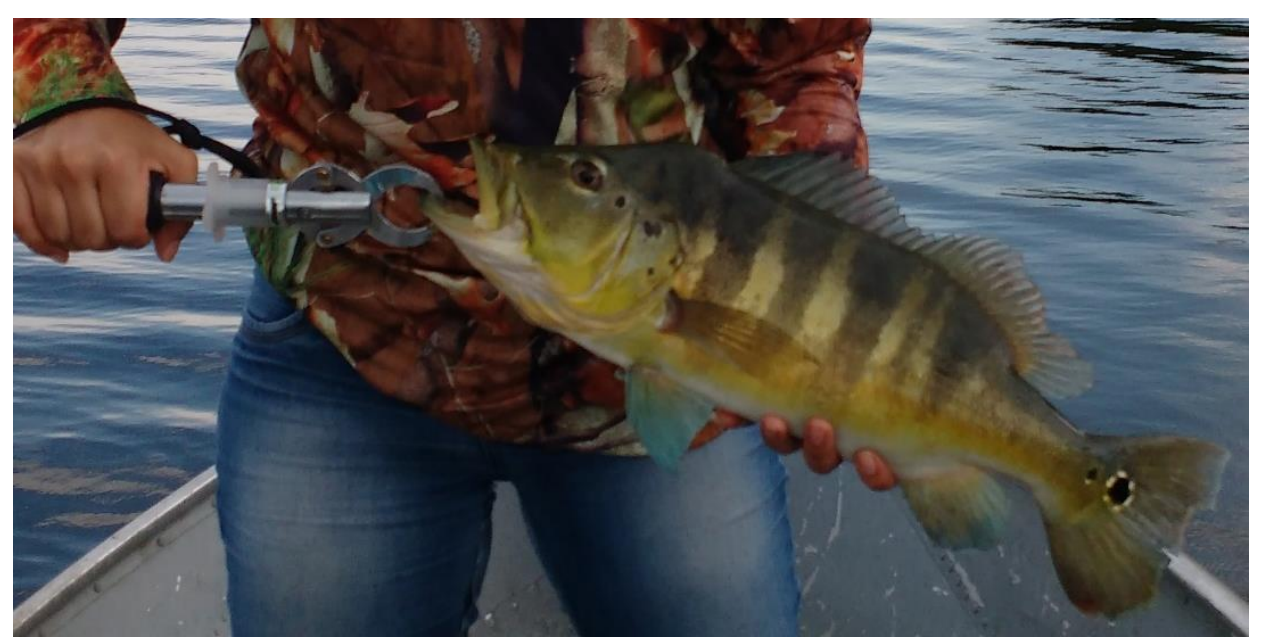

Figura 2. Tucunaré-azul capturado na região de Albuquerque, Corumbá-MS.

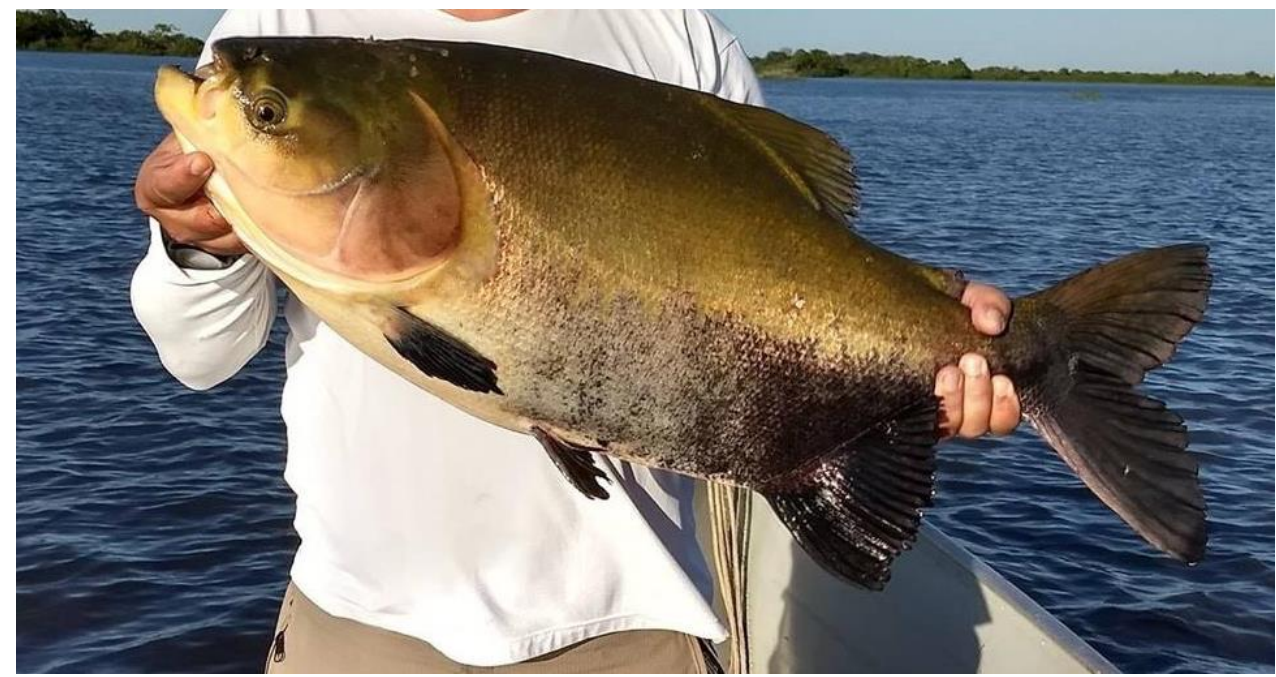

Figura 3. Tambaqui capturado na Baía Vermelha, rio Paraguai, Corumbá-MS.

\subsection{Possíveis impactos e pesca}

Sunaga e Verani (1997) e Pelicice e Agostinho (2009), em estudos abrangendo lagos do vale do Rio Doce e no reservatório Rosana, na Bacia do rio Paraná, respectivamente, relatam que o aumento da população de tucunarés é acompanhado de um decréscimo de espécies de peixes nativas. Em Rosana, nos anos de 2003 a 2007, foi detectada uma forte redução (95\%) na densidade de peixes nativos e $(80 \%)$ na riqueza de espécies dos peixes associados às macrófitas aquáticas (PELICICE e AGOSTINHO, 2009).

Em estudos feitos por Ferrareze e Nogueira (2015), também, no reservatório de Rosana, os resultados mostraram que tucunarés da espécie Cichla kelberi aparentam não ter impactado significativamente na ictiofauna local, conforme evidenciado por Pelicice e Agostinho (2009). Os resultados relatam que, após a introdução de Cichla kelberi no reservatório, valores associados à diversidade se apresentaram positivos. Os autores estimam que a introdução de um competidor favoreceu o aumento da abundância de espécie menores como Cyphocarax. modestus, Roeboides descalvadensis e Aphyocharax. anisitsi, entretanto não excluem a potencialidade da espécie de interferir na ictiofauna local ainda que não estabelecida. 
No Tocantins, com a recente implementação do reservatório da UHE Luís Eduardo Magalhães (Lajeado), a espécie Cichla piquiti ganhou evidência, adquirindo relevância econômica, tanto para a pesca artesanal, quanto para a pesca esportiva, desempenhando um papel importante na pesca local (PILICICE e AGOSTINHO, 2012). Machado e Costa (2017) apontam que existe uma carência na sistematização dos dados referentes ao segmento esportivo em Corumbá tendo por base os dados da Polícia Militar Ambiental que apontam somente os pescadores que procuraram o órgão para realizar a inspeção e legalização do pescado.

O tucunaré encontrou no Pantanal Sul Mato-Grossense características ambientais similares de seu nicho original e, por ser um peixe com alta capacidade adaptativa, sua introdução resulta em nítida preocupação devido a possíveis impactos negativos à ictiofauna nativa do Pantanal.

Takemoto e Pavanelli (1996), em trabalhos com ciclídeos na bacia do Paraná, registraram a introdução de novos parasitos relacionados ao gênero Cichla como Proteocephalus macrophallus e P. microscopicus. Machado et al. $(2000 ; 2005)$ registraram a presença do acantocéfalo Quadrigyrus machadoi e observaram altos índices de infestação nas espécies introduzidas em relação às nativas como também a ocorrência de metacercária Diplostomum (Austrodiplostomum) compactum nos olhos de exemplares da espécie Cichla monoculus, coletados na região.

O tambaqui, por sua vez, pode competir por recursos alimentares, territoriais e sexuais, pois é uma espécie que consegue produzir híbridos com o pacu: o tambacu (fêmea de tambaqui e macho de pacu) e paqui (fêmea de pacu e macho de tambaqui), processo que pode ocasionar uma perda genética gradativa na população do pacu pantaneiro (SARACURA e CASTAGNOLLI, 1990; RUBIDGE e TAYLOR, 2004).

Em cativeiro, esses híbridos (Figura 4) reúnem o melhor das duas espécies como maior resistência a baixas temperaturas da água, rusticidade e rápido crescimento, contudo na natureza essa hibridização pode desencadear perda da diversidade genética do pacu bem como sua diminuição populacional (ALVES et al., 2014). Tais fatos podem ser agravados quando se adiciona à pressão de pesca sob o pacu.

Por serem espécies de peixes forrageiros onívoros, pacu e tambaqui apresentam dentes que facilitam o esmagamento de frutas e sementes de revestimento duro e em sua dieta frutas carnosas e secas. Os Tambaquis estão associados com a dispersão de 76 espécies de plantas na Amazônia, podendo dispersá-las a quilômetros do seu local de origem (CORREA et al., 2015; ANDERSON et al., 2011). No Pantanal, o pacu é um peixe que dispersa algumas sementes de espécies vegetais de matas ciliares, evidenciando a dependência mútua entre peixes e plantas no ambiente pantaneiro (FURLAN, et al., 2017). Por terem hábitos alimentares semelhantes, a competição interespecífica por recursos pode aumentar e ocasionar impactos na estrutura populacional do pacu, além de afetar a dinâmica das interações com as plantas nativas na região (RODRIGUES, 2014; BRITSKI at al., 2007).

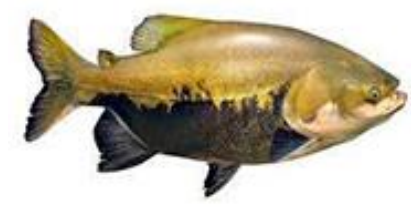

TAMBAQUI

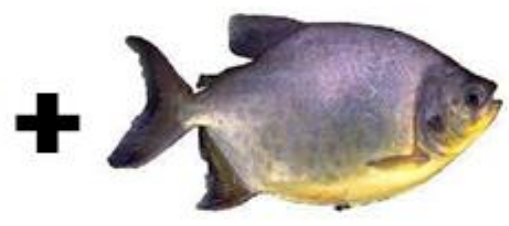

PACU

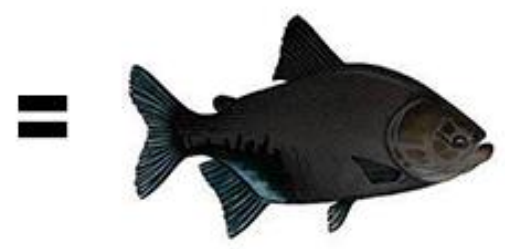

TAMBACU

Figura 4. Cruzamento entre o tambaqui fêmea e o pacu macho resultando no tambacu. Fonte: https://www.lauspesca.com/2019/01/dicas-de-pesca-diferenca-entre-tambaqui.html. Acesso: 21 abr 2020. 
Para Chellappa et al. (2003), tucunarés têm capacidade de adaptação fenotípica para alocação de recursos para crescimento ou reprodução, de acordo com mudanças nas condições ambientais, podendo assumir desova parcelada durante o ciclo reprodutivo anual, promovendo a explosão demográfica da população desses indivíduos e, consequentemente, aumentar a exploração dos recursos naturais e disponibilidade de outras espécies para a pesca comercial ou esportiva.

Para Catella (2003), a pesca realizada dentro dos limites naturais de reposição das populações se direciona ao excedente dos estoques e seu custo ambiental é nulo. Dessa forma, é possível também monitorar populações e o ambiente. Muito além de estabelecer uma cota de pescado específica para o tucunaré-azul e tambaqui, é importante que tal prática seja oficialmente estimulada por políticas públicas para manejo dessas espécies alóctones. O fomento da pesca dessas espécies deve contribuir tanto para o controle quanto para o monitoramento populacional dos respectivos peixes. A retirada de exemplares pode se tornar um caminho viável de controle populacional, e de acordo com a esportividade dessas espécies, pode promover a diversificação do setor pesqueiro na região pantaneira.

Não há dados suficientes para comprovar que o tucunaré e o tambaqui estão estabelecidos no Pantanal, pois há possibilidade de que os efeitos da introdução foram ou estão amortecidos pela plasticidade dos peixes ao longo do tempo (RUESINK, 2005). As estabilidades das espécies podem estar relacionadas com a competição alimentar, alto índice de canibalismo no caso do tucunaré e a extensa presença de macrófitas aquáticas nas baías e corixos (FERRAREZE e NOGUEIRA, 2015). Contudo quando um predador encontra a oportunidade de se espalhar, podem produzir mudanças pronunciadas na comunidade local e este evento não pode ser negligenciado (MCKINNEY, 2006).

A preocupação crescente com a preservação e conservação da natureza têm reflexos diretos nos campos sociais, econômicos e políticos no mundo todo (CAVALCANTI, 2012). De modo geral, conservar recursos naturais significa utilizá-los de maneira racional e sustentável, para que a pressão sobre eles não gere extinção ou depauperamento de espécies nativas. Por outro lado, o tucunaré e o tambaqui são espécies alienígenas que podem causar danos severos ao bioma Pantanal e se tornarem um problema ambiental como está sendo a carpa-capim (Ctenopharyngodon idella) e a carpa comum (Cyprinus carpio) que impactaram poderosamente os rios dos Estados Unidos levando à extinção espécies nativas e ocasionando um prejuízo estimado em 1 bilhão de dólares por ano na Flórida e 4,5 bilhões na região dos Grandes Lagos (TAYLOR et al.,1984; PEREZ et al., 2017; PIMENTEL, 2005).

\section{CONSIDERAÇÕES FINAIS}

Após mais de 30 anos das introduções do tucunaré e do tambaqui, algumas regiões tradicionais de pesca no Pantanal corumbaense já são conhecidas pela ocorrência dessas espécies. A pesca do tucunaré já é característica na região Cervo, (que está no rio Paraguai-mirim), Baía Albuquerque, Baía da Galinha, Baía Grande e Baía da Santa (Forte Coimbra), pois todos os anos pode-se encontrar tucunaré-azul nessas localidades. Já o tambaqui é anualmente encontrado na Baía vermelha, Baía Albuquerque e Baía da Santa (forte Coimbra). Híbridos entre tambaqui e pacu (tambacu) são encontrados em várias regiões do Pantanal corumbaense, contudo são mais dependentes do fluxo de cheia da região.

Há poucos estudos sobre essas espécies na região o que dificulta mensurar os impactos que elas proporcionam no bioma Pantanal. Por isso, é necessário desenvolver estudos para averiguar quais relações as espécies estão desempenhando na região, tanto com o ambiente quanto com as 
espécies nativas. Por serem espécies de interesse pesqueiro, principalmente por pescadores esportivos, a regulamentação de políticas específicas para elas pode proporcionar um ativo econômico atrativo para o mercado pesqueiro da região.

\section{REFERÊNCIAS}

ANDERSON, J.T.; NUTTLE, T.; ROJAS, J.S.S.; PENDERGAST, T.H.; FLECKER, A.S. Extremely longdistance seed dispersal by an overfished Amazonian frugivore. Proceeding of the Royal Society B Biological Sciences, v. 278, p. 3329-3335, 2011.

AGOSTINHO, A. A.; JÚLIO JÚNIOR, H. F. Peixes de outras águas: ameaça ecológica. Ciência Hoje, v. 21, n. 124, p. 36-44, 1996.

ALHO, C. J. R.; MAMEDE, S.; BITENCOURT, K.; BENITES, M. Introduced species in the Pantanal: implications for conservation. Brazilian Journal of Biology, v. 71, n. 1, p. 321-325, 2011.

ALHO, C. J. R. Efeitos do fluxo dos rios nos processos ecossitêmicos do Pantanal. In: ALVES, G. L.; Mercante, M. A.; FAVERO, S. (Org.) Pantanal Sul-Mato-Grossense - Ameaças e Propostas. Autores Associados, Campinas. 2012. 75 - 101 p.

ALVES, A. L.; VARELA, E. S.; MORO, G. V.; KIRSCHNIK, L. N. G. Riscos genéticos da produção de híbridos de peixes nativos. Embrapa Pesca e Aquicultura, Palmas, 2014. 60p. (Documentos, 03)

BRABO, M. F.; PEREIRA, L.F. S.; SANTANA, J. V. M.; CAMPELO, D. A. V.; VERAS, G. C. Cenário atual da produção de pescado no mundo, no Brasil e no estado do Pará: ênfase na aquicultura. Acta of Fisheries and Aquatic Resources, v. 4, n. 2, p. 50-58, 2016.

BRAZIL, M. C - Rio Paraguai - O "Mar Interno" Brasileiro. Ed UFMS, Campo Grande, 2014. $366 \mathrm{p}$.

BRITSKI, H.A; SILIMON, K. Z. S.; LOPES, B. S. Peixes do Pantanal: manual de identificação. 2 ed. Brasília: Embrapa Informação Tecnológica. 2007. 230 p.

BRITSKI, H. A. Sistemática dos peixes de água doce e sua importância nos projetos do setor elétrico. In: COMASE - Seminário sobre fauna aquática e o setor elétrico brasileiro. Caderno 1 Fundamentos, 1994, Rio de Janeiro. Anais do Seminário sobre fauna aquática e setor elétrico brasileiro. Rio de Janeiro: UERJ, 1994. p. 23-30.

BUCKUP, P. A.; MENEZES, N. A.; GHAZZI, M. S. A. Catálogo das espécies de peixes de água doce do Brasil, v1. Rio de Janeiro: Museu Nacional, 2007. 195 p.

CARVALHO, N. de O. Hidrologia da bacia do alto Paraguai. In: Simpósio sobre recursos naturais e socioeconômicos do pantanal. 1986, CORUMBÁ. Anais do Simpósio sobre recursos naturais e socioeconômicos do pantanal. Brasília: Embrapa-DDT, 1986. p. 43-29. 
CATELlA, A. C. A Pesca no Pantanal Sul: situação atual e perspectivas. Corumbá: Embrapa Pantanal, 2003. 45 p. (Documentos, n. 48)

CAVALCANTI, C. Sustentabilidade: mantra ou escolha moral? Uma abordagem ecológicoeconômica. Estudos avançados, v. 26, p. 34-50, 2012.

CÓRDOVA JUNIOR, R. F. Da S. A ocorrência e pesca do tucunaré azul no Pantanal Sul-MatoGrossense. In SILVA, M. E. D (Org.). Desafios Teóricos e Aplicados da Ecologia Contemporânea 2. Atena Editora, Ponta Grossa, 2020. 88 p.

CORREA, S.B.; COSTA-PEREIRA, R.; FLEMING, T., GOULDING, M.; ANDERSON, J.T. Neotropical fish-fruit interactions: eco-evolutionary dynamics and conservation. Biological Reviews of the Cambridge Philosophical Society, v. 90, n. 4, p. 1263-1278, 2015.

CHELlAPPA, S.; CÂMARA, M. R.; CHEllaPPA, N. T.; BEVERIDGE, M. C. M.; HUNTINGFORD, F. A. Reproductive ecology of a neotropical cichlid fish, Cihla monoculus (Osteichthyes: Cichlidae). Brazilian Journal of Biology, v. 63, n. 1, p.17-26, 2003.

FARIAS, I.P.; ORTI G.; SAMPAIO I.; SCHNEIDER, H.; MEYER, A. Mitochondrial DNA phylogeny of the family Cichlidae: Monophyly and fast molecular evolution of the neotropical assemblage. Journal of Molecular Evolution, v.48, n6. 1999. p.703-711

FERRAREZE, M.; NOGUEIRA, M.G. Impact assessment of the introduction of Cichla kelberi in a large Neotropical reservoir and its lateral lagoons (Upper Paraná River Basin, Brazil). Brazilian Journal of Biology, v. 75, n. 4, p.1018-1026, 2015.

FURLAN, A. O.; MUNIZ, C. C.; CARNIELLO, M. A. Análise do componente vegetal na alimentação de peixes e da relação com a dispersão de sementes no Pantanal Mato-Grossense. Revista Brasileira de Ciências Ambientais, n. 45, p. 61-70, 2017.

GALDINO, S.; VIEIRA, L.M.; OLIVEIRA, H.; CARDOSO, E.L. Impactos da agropecuária nos planaltos sobre o regime hidrológico do Pantanal. EMBRAPACPAP. 2002, 6 p. (Circular Técnica, 37)

GOMEIRO, L. M. Métodos de coleta utilizados na captura de tucunarés (Cichla spp.) para fins científicos. Revista Brasileira de Engenharia de Pesca, v. 5, n. 1, p. 1-13, 2010.

JEPSEN, D.B.; WINEMILLER, K.O.; TAPHORN, D.C. Temporal patterns of resource partitioning among Cichla species in a Venezuelan blackwater river. Journal of Fish Biology, v. 51, p. 1085-1108, 1997.

KULLANDER, S.; FERREIRA, E. A review of the South American cichlid genus Cichla, with descriptions of nine new species (Teleostei: Cichlidae). Ichthyological Exploration of Freshwaters, v. 17, n. 4, p. 289-398, 2006. 
LIPPARELLI, T. História natural do tucunaré Cichla cf. ocellaris (Teleostei, Cichlidae) no rio Piquiri, Pantanal de Paiaguás, Estado de Mato Grosso do Sul. 1999. 296p. Dissertação (Mestrado em Ciências Biológicas) - Universidade Paulista Júlio de Mesquita, Rio Claro, SP.

MACCORD, P.F.L.; SILVANO, R.A.M..; RAMIRES, M.S.; CLAUZET, M.; BEGOSSI, A. Dynamics of artisanal fisheries in two Brazilian Amazonian reserves: implications to comanagement. Hydrobiologia, v. 583, p. 365-376, 2007.

MACHADO, R. X.; COSTA, E. A. O turismo de pesca em Corumbá, na fronteira Oeste do Brasil. Revista Iberoamericana de Turismo- RITUR, v. 7, n. 1, p. 36-48, 2017.

MACHADO,P.M.; ALMEIDA,S.C.; PAVANELLI,G.C. Ecological aspects of endohelminths parasitizing Cichla monoculus Spix, 1831 (Perciformes: Cichlidae) in the Paraná River near Porto Rico, State of Paraná, Brazil. Comparative Parasitology, v. 67, n. 2, p. 210-217, 2000.

MACHADO, P.M.; TAKEMOTO, R.M.; PAVANELLI, G.C. Diplostomum (Austrodiplostomum) compactum (Lutz, 1928) (Platyhelminthes, Digenea) metacercaria in fish from the floodplain of the Upper Paraná River, Brazil. Parasitology Research, v.97, p.436-444, 2005 .

MARIANI, M. A. P.; ARRUDA D. O. Empreendimento de economia solidária da cidade de Corumbá/MS e suas relações com o grupo de pescadores artesanais urbanos, com vistas ao desenvolvimento local. Revista Acadêmica Observatório de Inovação do Turismo, v. 4, n. 2, p. $1-1,2009$.

MARQUES, D. K. S.; RESENDE, E. K. Distribuição do Tucunaré Cichla cf. monoculus (Osteichthyes, Cichlidae) no Pantanal. 2005. 28p. (Boletim de Pesquisa e Desenvolvimento, 60)

MCKINNEY, M.L. Urbanization as a major cause of biotic homogenization. Biological Conservation, v. 127, n. 3, p. 247-260, 2006.

ORSI, M. L.; AGOSTINHO, A. A. Introdução de espécies de peixes por escapes acidentais de tanques de cultivo em rios da Bacia do Rio Paraná, Brasil. Revista Brasileira de Zoologia, v. 16, n. 2, p.557-560, 1999.

PELICICE, F. M., AGOSTINHO, A. A. Fish fauna destruction after the introduction of a nonnative predator (Cichla kelberi) in a Neotropical reservoir. Biologial Invasions, v. 11, p. 17891801, 2009.

PELICICE, F. M.; AGOSTINHO, C. S. Deficient downstream passage through fish ladders: the case of Peixe Angical Dam, Tocantins River, Brazil. Neotropical Ichthyology, v. 10, n. 4, p. 1-10, 2012.

PENNA, M. A. H.; VILLACORTA-CORRÊA, M. A.; WALTER, T.; PETRERE JUNIOR, M. Growth of the tambaqui Colossoma macropomum (Cuvier) (Characiformes: Characidae): which is the best model? Brazilian Journal of Biology, v. 65, n. 1, p. 129-139, 2005. 
PEREZ, C. A. R.; WAICHEIM, M. A.; CORDERO, P.; BLASETTI, G. Qué se esconde tras la introducción de especie? La invasión de la carpa común en Patagonia. Desde la Patagonia, v. 14. n. 3, p. 36-41, 2017.

PIMENTEL, D. Aquatic nuisance species in the New York State Canal and Hudson River systems and the Great Lakes Basin: an economic and environmental assessment. Environmental Management, v. 35, p. 692-702, 2005.

RESENDE, E. K.; MARQUES, D. K. S.; FERREIRA, L. K. S. G. A successful case of biological invasion: the fish Cichla piquiti, an Amazonian species introduced into the Pantanal, Brazil. Revista Brasileira de Biologia, v. 68, n. 4, p. 799-804, 2008.

RUESINK, J.L. Global analysis of factors affecting the outcome of freshwater fish introductions. Conservation Biology, v. 19, n. 6, p. 1883-1893, 2005.

RODRIGUES, A. P. O. 2014. Nutrição e alimentação do tambaqui (Colossoma macropomum). Boletim do Instituto de Pesca, v. 40, n.1, p. 135-145, 2014.

ROSENFIELD, A.; MANN, R. Dispersal of living organismo into aquatic ecossystems. Maryland Sea Grant Publications. 1992. 471 p.

RUBIDGE, E. M.; TAYLOR, E. B. Hybridization zone structure and the potential role of selection in hybridizing populations of native westlope cutthroat trout (Oncorhynchus clarki lewisi) and introduced rainbow trout (O.mykiss). Molecular Ecology, v. 13, n. 12, p. 3725-3749, 2004.

SABINO, J.; ZUANON, J. A. S. A stream fish assemblage in Central Amazonia: distribution, activity patterns and feeding behavior. Ichthyological Exploration of Freshwaters, v. 8, n. 3, p. 201-210, 1998.

SABINO, J. Pantanal e seus encantos. In: HANNIBAL, W.; DUARTE, L. A.; CAVAlC, C. Mamíferos não-voadores do Pantanal e entorno. Natureza em Foco, 2015. p. 14-33.

SARACURA, V. F.; CASTAGNOLLI, N. Comparação do desempenho entre alevinos de Pacu (Piaractus mesopotamicus) e híbridos de Pacu e Tambaqui (Colosoma macropomum). Ciência Zootécnica, v. 5, n. 3, p. 17-19,1990.

SILVA, J. W. B.; CHACON, J. O.; SANTOS, E. P. Curva de rendimento do tucunare pinima Cichla temensis (Humboldt, 1833), do açude público "Estevam Marinho" (Curemas, Paraíba, Brasil) (Pisces, Actinopterygii, Cichlidae). Revista Brasileira de Biologia, n. 40, p.203-206, 1980.

SIMBERLOFF, D. Confronting introduced species: a form of xenophobia? Biological Invasions, v. 5, p. 179-192. 2003.

SOARES, M. G. M. Peixes de lagos do Médio Rio Solimões. 2 ed. Manaus: Instituto I-Piatam, 2007. 160p. 
SÚAREZ, I. R., CATELlA, A. C.; NASCIMENTO, F. L. Alimentação do Tucunaré Cichla sp. (PISCES, CICHLIDAE) um peixe amazônico introduzido no Pantanal, Brasil. Embrapa Pantanal. 2001. 17 p. (Boletim de Pesquisa, n. 24)

SUNAGA, T.; VERANI, J. R. The fish communities of lakes. In: TUNDISIM J. G.; SAIJO Y. Limnological studies on the rio Doce Valley lakes, Brazil. Academia Brasileira de Ciências. 1997. 528p.

TAYLOR, J. N. W. R.; COURTENAY, J. A.; MCCANN, A. Known impacts of exotic fishes in the continental United States. In W. R. COURTENAY; J. R. STAUFFER, editors. Distribution, biology, and management of exotic fishes. Johns Hopkins University Press, 1984. p. 322- 373.

TAKEMOTO,R.M.; G.C.PAVANELLI. Proteocephalidean Cestodes in the Freshwater Fish, Cichla monoculus from the Parana river, Brazil. Stud. Neotropical Fauna \& Environment, v. 31 p. 123-127, 1996.

VELLUDO, M. R.: Ecologia trófica da comunidade de peixes do reservatório de Cachoeira Dourada, rio Paranaíba, bacia do alto rio Paraná, Brasil. 2011. 123p. Tese (Doutorado) Universidade Federal de São Carlos.

VILA da SILVA, J.S. 1995. Elementos fisiográficos para delimitação do ecossistema Pantanal: Discussão e proposta, p. 439-458. In F.A. Esteves (ed.) Oecologia Brasiliensis, Estrutura, Funcionamento e Manejo de Ecossistemas Brasileiros. PPGEUFRJ, Rio de Janeiro, Brazil

WINEMILLER, K. O. Ecology of peacock cichlids (Cichla spp.) in Venezuela. Journal of Aquaculture and Aquatic Sciences, n. 9, p. 93-112, 2001. 